\title{
Regulación circadiana, patrón horario de alimentación y sueño: Enfoque en el problema de obesidad
}

\section{Circadian rhythms, eating patterns, and sleep: A focus on obesity}

\section{RESUMEN}

El reloj biológico determina la mantención de los ritmos circadianos en mamíferos, un tipo particular de ritmos biológicos de duración cercana a 24 horas. Existe una estrecha relación entre el funcionamiento del sistema circadiano, la alimentación y la regulación metabólica, lo que actualmente constituye un área de intensa investigación. En particular, la alteración de la ritmicidad circadiana a partir de modificaciones genéticas, conductuales o dietarias, lleva a trastornos comportamentales, ganancia de peso excesiva y alteraciones metabólicas. Algunos factores que contribuyen a la alteración o desajuste circadiano incluyen el jet-lag, el trabajo por turnos horarios, la desorganización temporal y restricción de sueño, y desorden del patrón horario de alimentación. Este trabajo resume la evidencia acerca de la influencia de los ritmos circadianos en procesos relacionados con la alimentación y las consecuencias metabólicas de su alteración. Se hace énfasis en las consecuencias de la alteración de los ritmos de alimentación-ayuno y de sueñovigilia, y su relación con la ganancia de peso excesiva, la obesidad y trastornos metabólicos asociados, condiciones altamente prevalentes en sociedades occidentalizadas. Palabras clave: Ritmos circadianos; Alimentación; Patrón horario alimentario; Obesidad; Sueño; Trabajo por turnos.

\footnotetext{
ABSTRACT

In mammals, the biological clock is driven by circadian rhythms, a particular type of biological rhythm that last about 24 hours. There is a close relationship between the functioning of the circadian system, eating and metabolic regulation, which is currently an area of intense research. Alteration of circadian rhythmicity from genetic, behavioral or dietary modifications, leads to behavioral and metabolic disorders, and excessive weight gain. Factors that contribute to circadian disruption include, among others, jet lag, shift work, mistimed and restricted sleep, and irregular eating patterns. This review summarizes the evidence regarding the influence of circadian rhythms on eating processes and the metabolic consequences of circadian disruption. Special focus is on the consequences of disruption of regular eatingfasting and sleep-wake rhythms, and relationships with
}

Rodrigo Chamorro ${ }^{1}$, Rut Farías ${ }^{2}$, Patricio Peirano ${ }^{3}$.

1. Departamento de Nutrición, Facultad de Medicina, Universidad de Chile. Santiago, Chile. 2. Escuela de Nutrición y Dietética, Facultad de Medicina, Universidad de Chile. Santiago, Chile. 3. Laboratorio de Sueño, Instituto de Nutrición y Tecnología de los Alimentos, Universidad de Chile. Santiago, Chile.

Dirigir correspondencia a: Rodrigo Chamorro. Departamento de Nutrición, Facultad de Medicina, Universidad de Chile. Av. Independencia 1027, Independencia, Santiago, Chile. Telefono: $+562-229786420$.

E-mail: rodrigochamorro@med.uchile.cl

Este trabajo fue recibido el 12 de diciembre de 2017. Aceptado con modificaciones: 05 de enero de 2018. Aceptado para ser publicado: 23 de enero de 2018.

excessive weight gain, obesity and obesity-related metabolic disorders that are highly prevalent in westernized societies. Key words: Circadian rhythms; Eating pattern; Obesity; Sleep; Shift work.

\section{INTRODUCCIÓN}

Los ritmos biológicos son adaptaciones de los organismos vivos a su medio ambiente que influencian a las más diversas funciones biológicas. Un tipo particular de ritmos biológicos lo constituyen los ritmos circadianos (RC) y un claro ejemplo de su influencia lo constituye el ciclo de actividad-reposo, el ciclo sueño-vigilia (CSV) o el perfil de secreción de diversas hormonas a través de las 24 horas. Actualmente, se reconoce a un Sistema Circadiano, 
organización jerárquica conectada a nivel central y periférico.

Por su parte, la regulación de la ingesta alimentaria responde a factores homeostáticos, hedónicos y sociales pero también circadianos, todos los que contribuyen al patrón de alimentación (PA) habitual'. La nutrición, la alimentación y las respuestas metabólicas a la ingesta de alimentos son reguladas por los RC y también por variaciones diurnas a través del día. Es de esperar entonces que un patrón de alimentación-ayuno relativamente estable influya positivamente en una adecuada regulación circadiana y metabólica. De hecho, como se resalta en esta revisión, reciente investigación sustenta un efecto importante de la alimentación, como un factor sincronizador del sistema circadiano $^{2}$. Este artículo se centra en la influencia de los RC sobre la nutrición y alimentación. Especial énfasis se pone en describir el sistema circadiano, el rol de la alimentación como factor regulador del mismo, la importancia del momento o timing de la ingesta alimentaria a través de las 24 horas, así como en las consecuencias de la alteración circadiana que ocurre, por ejemplo, en quienes trabajan en modalidad de turnos.

\section{Ritmos circadianos y crononutrición}

Los ritmos biológicos son adaptaciones de los organismos a su medio ambiente y caracterizan las funciones de nuestro organismo, contribuyendo a una mejor adaptación y mantención de un orden temporal interno ${ }^{3}$. Los RC (del latín circa y diem, alrededor de un día), se caracterizan por tener duración aproximada de 24-horas, período en el cual se presenta una variación entre el máximo y el mínimo de actividad en diversas variables o funciones biológicas estudiadas 4 .

En mamíferos, el reloj biológico o pacemaker central está ubicado en el núcleo supraquiasmático (NSQ) hipotalámico, el que comanda y regula los diversos $\mathrm{RC}^{5}$. Su relevancia radica en que confiere al sistema nervioso central (SNC) la capacidad de sincronizar e integrar nuestra fisiología antes de que ocurran los predecibles cambios medioambientales biológicamente relevantes, como la alternancia luz-oscuridad, la disponibilidad de alimento, cambios de temperatura o humedad ${ }^{4}$. A nivel molecular, los RC son generados por los denominados genes reloj, conjunto de genes que conforma un mecanismo de transcripción que involucra loops de retroalimentación positiva y negativa; entre estos se incluyen Clock, Bmal1, Per (1, 2 y 3), Cry (1 y 2), Rev-erba, Ror-a ${ }^{6}$; estos regulan a su vez a los denominados genes controlados por el reloj, muchos de ellos relacionados directamente con vías enzimáticas y metabólicas, constituyendo un mecanismo de interacción entre el funcionamiento del reloj circadiano y las funciones metabólicas ${ }^{7}$.

A nivel sistémico la ritmicidad circadiana se organiza a través del denominado Sistema Circadiano (Figura 1). Este es comandado a nivel central por el NSQ que sensa la intensidad del estímulo de luz (la luz es el estímulo sincronizador más importante), a través de su comunicación con neuronas fotosensibles de la retina (que expresan melanopsina) ${ }^{8}$. El
NSQ entonces sincroniza diariamente a diversos relojes circadianos localizados tanto dentro como fuera del SNC, por ejemplo en órganos como hígado, tejido adiposo, glándulas suprarrenales, páncreas, riñón, corazón y músculo esquelético ${ }^{9}$. Así como la alternancia de luz/oscuridad es el principal estímulo sincronizador para el NSQ, la ingesta de alimentos es también un estímulo sincronizador potente para los osciladores periféricos ${ }^{7}$. Incluso, se ha sugerido que el estímulo de luz podría constituir un estímulo sincronizador para los osciladores periféricos, en forma independiente del $\mathrm{NSQ}^{10}$. La concepción predominante es que el sistema circadiano es una organización vertical, jerárquica y consecuentemente el NSQ sincroniza a los relojes u osciladores periféricos día a día. Además, se ha propuesto recientemente un modelo federado de regulación, en el que los osciladores periféricos tendrían la capacidad de sincronización celular a nivel intra-organo así como inter-organos, lo que favorecería la capacidad del organismo de mantener sincronía tanto interna como con su medio ambiente ${ }^{10}$.

Existe una estrecha interacción entre diversos procesos metabólicos y la ritmicidad circadiana ${ }^{11}$. La actividad enzimática y hormonal, el metabolismo de nutrientes, la temperatura corporal, presión arterial y el ciclo sueño-vigilia (CSV), son todos procesos regulados por los $\mathrm{RC}^{3}$, tal como lo son la sensación de apetito/hambre ${ }^{12}$, funcionalidad del tracto gastrointestinal, síntesis y absorción de ácidos biliares y la motilidad intestinal ${ }^{13}$. Lo anterior es importante considerando que las consecuencias metabólicas e incluso la sintomatología postprandial, se ven influidas por la hora del día en que ocurre la alimentación.

Un aspecto de especial interés en la interacción entre el sistema circadiano y la nutrición, es el rol que tiene la alimentación como factor sincronizador relevante de los $\mathrm{RC}^{14}$. Tocante a la dimensión circadiana en los procesos nutricionales, la Crononutrición hace referencia el estudio de la interacción entre la alimentación, la nutrición y el reloj circadiano; específicamente, al estudio de los efectos del timing y la composición de la ingesta alimentaria (energía y macronutrientes) a través del día, sobre la organización del sistema circadiano, concebidos estos en una modalidad de interacción recíproca o bidireccional ${ }^{2}$.

\section{Influencia circadiana en el patrón de alimentación}

EI PA se refiere a las características y organización horaria de las comidas que un individuo realiza diaria y habitualmente, incluyendo el número (o frecuencia alimentaria), tipo (principales, colaciones, extras), regularidad horaria (omisión o constancia), composición (aporte de energía y nutrientes) y el contexto en que ocurre la alimentación (en el hogar, en compañía, otros) ${ }^{15}$. En la regulación del PA habitual intervienen factores homeostáticos, hedónicos, sociales, circadianos y ultradianos' ${ }^{1}$. El PA en el humano es diurno caracterizado por la secuencia ininterrumpida de episodios de ingesta (comidas) e intervalos de ayuno. Se ha descrito la frecuencia de tres comidas principales (típicamente 
desayuno, almuerzo y cena) como un patrón mantenido a lo largo del tiempo en diversas poblaciones ${ }^{3}$; ello, junto con el hecho de que dicho patrón es también evidente en individuos aislados de estímulos medioambientales, sugieren la influencia del reloj circadiano en $\mathrm{el}^{3}$. Los RC influencian además los componentes homeostáticos y hedónicos de la ingesta alimentaria, aún cuando este último aspecto ha sido menos estudiado ${ }^{16}$.

EI PA ha sufrido cambios evidentes en sociedades occidentalizadas, por ejemplo, relacionado al número de comidas, horarios y regularidad de las mismas. Así por ejemplo, en la década de 1960 el consumo de desayuno diario en adolescentes estadounidenses mostraba una prevalencia en torno al $90 \%$, la que se redujo cercano al $70 \%$ luego de 3 décadas ${ }^{17}$. Algunos reportes muestran que actualmente alrededor del $10-30 \%$ de los adolescentes no desayuna en forma diaria, siendo algunos de los factores asociados un ritmo de vida más acelerado, acortamiento de las horas destinadas a dormir en la noche, y la ausencia de patrones familiares estructurados ${ }^{17}$. A partir de estudios observacionales, se reconoce un efecto positivo de la ingesta regular del desayuno para una alimentación y estado nutricional saludable y disminución del riesgo de obesidad $^{18}$, sin embargo, estudios experimentales no respaldan necesariamente esa relación ${ }^{19}$. Si se ha mostrado, por ejemplo, que un desayuno (>700 kcal/día) diario durante 6 semanas, resulta en mayor termogénesis inducida por actividad física y mejor regulación de la glicemia durante la tarde y noche ${ }^{20}$. $\mathrm{Si}$ bien la evidencia experimental no respalda todos los efectos saludables atribuibles al desayuno per se, sí apoya su importancia para una adecuada regulación metabólica.

Igualmente, la frecuencia alimentaria (o el número de comidas al día) sería relevante para la regulación del metabolismo y del peso corporal, y se asocia inversamente con el peso corporal y la presencia de sobrepeso ${ }^{21}$. En un estudio experimental, Stote y cols. ${ }^{22}$, compararon el efecto del consumo de 1 ó 3 comidas por día durante 8 semanas (con idéntica ingesta calórica total), y mostraron mayor sensación de hambre y alteración del perfil lipídico luego de 1 comida/día, a pesar de no observar cambios en la composición corporal ${ }^{22}$; a su vez, la irregularidad de las comidas (ej. 3 - 9 comidas/día vs. 6 comidas/día a horario fijo durante 2 semanas) se relaciona con hallazgos similares ${ }^{23}$.

Aún cuando la propuesta de aumentar el número de comidas es promovida como un PA deseable para reducir el peso coporal y algunas alteraciones metabólicas, la evidencia experimental no lo sustenta. En efecto, Leidy y cols. ${ }^{24}$ en una revisión de estudios experimentales con alimentación controlada, concluyeron una ausencia de (o muy leve) efecto de una mayor frecuencia de comidas ( $>3$ / día) sobre la regulación de ingesta de alimentos o el control del apetito ${ }^{24}$. Aún más, la mayor frecuencia de comidas/día podría facilitar un sobreconsumo de energía y nutrientes y ganancia de peso excesiva derivada de mayores ocasiones para comer ${ }^{24}$; aún cuando, podría influir positivamente en la baja de peso bajo condiciones de restricción calórica pero no así con un PA habitual25.

Resulta interesante que el momento en que las comidas ocurren a través de las 24 horas parece tener un rol tan o más importante que el número de comidas/día o que el total de calorías ingeridas ${ }^{26}$, en el contexto de la regulación energética en humanos. Si bien la mantención de 3 comidas principales a lo largo del día se ha propuesto como parte importante de una alimentación saludable y como modelo a nivel poblacional, más recientemente se ha cuestionado, proponiendo otra estructura temporal más ajustada a nuestra fisiología en un contexto circadiano ${ }^{27}$. Entre otras, se propone ajustar (o restringir, ver más abajo) la ingesta alimentaria al período diurno con el consumo de 2 ó 3 comidas principales exclusivamente durante el período de luz, 3 comidas pequeñas en mismo período o la alternancia de comidas con períodos de ayuno intermitente ${ }^{27}$. Además, un estudio reciente mostró que el momento en que se consumen carbohidratos dentro de una comida principal (ej. almuerzo) modifica la respuesta metabólica a la comida en pacientes con diabetes tipo 2 (DT2), presentando menor incremento de glucosa e insulina a los 180', cuando estos fueron consumidos al final de la misma ${ }^{28}$, demostrando que el timing de la alimentación sería clave a lo largo del día pero también en una comida particular.

\section{La ingesta de alimentos y el sistema circadiano}

Diversos estudios en roedores han mostrado que el RC de expresión de los genes reloj en tejidos periféricos es influenciado fuertemente por la ingesta alimentaria. Damiola y cols. ${ }^{29}$, describieron que la restricción de la disponibilidad de alimento al período diurno (período habitual de sueño) por 9 días, invirtió la fase de expresión génica (hasta en 12 horas) de los osciladores periféricos (en hígado, riñón páncreas y corazón) pero no modificó la fase de expresión en el NSQ; lo anterior, sugiere que la restricción de alimentación a través del día, ejerce un efecto potente para la sincronización de los osciladores circadianos periféricos sin afectar el oscilador central, incluso en ausencia de estímulo lumínico ${ }^{29}$.

De igual manera, la llamada actividad anticipatoria al alimento, ilustra el rol de la alimentación en la organización circadiana. Este fenómeno descrito hace décadas en roedores mantenidos en un marco temporal de 24-horas, incluye cambios conductuales y fisiológicos anticipatorios a la llegada del alimento cuando este se restringe, generando un estado catabólico. La influencia circadiana es bien reconocida en este fenómeno, pues se identifica aún en condiciones ambientales constantes y desaparece si las comidas se entregan en un período mayor de 24 horas $^{30}$; no obstante, aún no se ha identificado el centro regulador (u oscilador) de esta actividad, la cual persiste incluso en ausencia de un NSQ funcional ${ }^{31}$.

Otra aproximación al rol del alimento como factor regulador de los RC, proviene de estudios que han modificado el momento de disponibilidad del alimento durante el día y evaluado sus efectos metabólicos y fisiológicos. Arble y 
cols. ${ }^{32}$, mostraron que ratas alimentadas exclusivamente durante su período habitual de reposo (diurno), tuvieron una ganancia de peso excesiva desde la segunda semana, en comparación a animales alimentados durante el período nocturno, a pesar de tener ingesta calórica y actividad física diaria similar ${ }^{32}$. También se había mostrado que recibir sólo una comida/día hacia el final del período de actividad/vigilia (nocturno) se manifiesta en aumento de peso, alteración de la tolerancia a la glucosa y dislipidemia ${ }^{33}$, datos que en conjunto, sustentan la relevancia del momento en que ocurre la alimentación sobre la homeostasis energética.

Por su parte, la disminución o restricción del tiempo de disponibilidad de alimentos (time-restricted feeding) también tiene consecuencias sobre el sistema circadiano. Ratones a los que se reduce el tiempo que disponen de alimentos mejoran su regulación metabólica tanto en el período de luz como de oscuridad, en comparación con los que tienen acceso ad-libitum ${ }^{34}$, hallazgos concordantes con otros que muestran que la restricción de una dieta rica en grasa a $8 \mathrm{~h} /$ día exclusivamente durante el período de oscuridad, previene la ganancia de peso excesiva, hyperinsulinemia, esteatosis hepática, y alteración de la expresión de los genes reloj ${ }^{14}$.

Estudios clínicos en adultos con sobrepeso, obesidad y en patologías como DT2 $2^{35,36}$, ya han demostrado el efecto benéfico de la modificación temporal de la alimentación sobre la regulación del metabolismo de glucosa y baja de peso. Interesantemente, estudios recientes también dan cuenta del efecto metabólico de la restricción del tiempo de disponinilidad de alimentos. Un protocolo con alimentación restringida a 8 horas/día/2 meses, resultó en una reducción de la grasa corporal y menor cuociente respiratorio sin modificación de la masa muscular en sujetos entrenados ${ }^{37}$.

Sin duda que una mejor comprensión del timing de alimentación y sus consecuencias metabólicas con propósitos preventivos y eventualmente terapéuticos, sería particularmente útil en pacientes con obesidad y en grupos específicos expuestos a un desajuste circadiano, como quienes trabajan en modalidad de turnos horarios.

\section{Cronoalteración \\ I. Alteración del patrón de alimentación}

Algunos de los cambios cuanti- y cualitativos anteriormente descritos relacionados al $\mathrm{PA}^{38} \mathrm{y}$ al $\mathrm{CSV}^{39}$ en sociedades occidentalizadas, incidirían en la desorganización de los RC y sus consecuencias metabólicas ${ }^{40}$. Por ejemplo, la proporción de adultos Estadounidenses que consumían regularmente 3 comidas/día ha disminuido cercana al $60 \%$ en ambos sexos al año 2010, mientras que la proporción de energía a partir de snacks o colaciones ha aumentado cercano al $25 \%$ en distintos grupos ${ }^{41}$. Datos interesantes en adultos de la misma población indican que el PA habitual dista mucho de aquel de 3 comidas principales; Gill y cols. ${ }^{26}$, confirmaron que gran parte (> 35\%) de la ingesta calórica ocurre después de las 18:00h y una gran variabilidad en la realización de las comidas, las que se organizan en forma dispersa (no en tres comidas principales) y con una duración aproximada del período en que estas ocurren de 14h.

Esos datos, corroborados en otras poblaciones ${ }^{42}$, indican que el PA se ha "desorganizado" a través de las 24 horas llevando a una disminución del tiempo esperable destinado al ayuno nocturno. Se ha descrito que adultos "dormidores tardíos" (aquellos individuos cuya mitad del período de sueño ocurrió pasadas las 05:30 a.m.), presentan elevada ingesta calórica ( $>50 \%$ del total de calorías/día) después de las 08:00 pm y una deficiente calidad dietaria ${ }^{43}$. A su vez, mayor riesgo de obesidad caracteriza a escolares ( 6 a 11 años) y adultos (21 a 69 años) ${ }^{44}$, que consumen más de un tercio de las calorías totales después de las 04:00 pm. Lo anterior es relevante ya que la apetencia por alimentos grasos aumenta en el horario de la tarde/noche ${ }^{3,45}$, lo que en conjunto con una alimentación con horarios más tardíos, constituyen factores de incremento de la ganancia de peso (Figura 1). En forma bastante reciente, McHill y cols. ${ }^{46}$, confirmaron que la alimentación tardía, se relaciona con mayor adiposidad en adultos con alto porcentaje de grasa corporal, independiente de la ingesta calórica total o el nivel de actividad física.

Estudios experimentales en mujeres obesas bajo una restricción dietaria de $1400 \mathrm{kcal} /$ día y que consumieron su aporte calórico con disminución progresiva desde la mañana hasta la noche $(700,500$, y $200 \mathrm{kcal}$, en desayuno, almuerzo y cena, respectivamente), tuvieron una mayor baja de peso y mejor control glicémico luego de 3 meses de intervención ${ }^{47}$, datos consistentes con aquellos en pacientes con DT $2^{36}$. Estudios epidemiológicos en adultos han descrito que una mayor proporción de carbohidratos (y menos de grasa) en las comidas de desayuno y almuerzo, se asoció a menor incidencia de DT2 y a un menor riesgo de presentar la patología en un período de 10 años $^{48}$, consistentes con otros que han mostrado que el consumo de consumo de carbohidratos después de las 20:00 h se asocia a mayor IMC ${ }^{49}$.

En adultos bajo una restricción calórica $(1500 \mathrm{kcal} /$ día), un desayuno rico en proteínas y carbohidratos resultó beneficioso para la mantención del peso luego de haberlo perdido, potenciando la baja de peso, la sensación de saciedad y la supresión de la hormona grelina, estimuladora de la apetencia ${ }^{50}$; existen escasos reportes contradictorios mostrando un efecto similar con mayor proporción de carbohidratos consumidos preferentemente en la cena ${ }^{51}$. Un estudio experimental bastante reciente mostró que un PA con comidas ricas en grasas en la mañana pero ricas en carbohidratos en la tarde-noche, resultó en mayor deterioro del metabolismo de la glucosa comparado con comidas con la composición inversa en sujetos con tolerancia a la glucosa alterada (pero no diabéticos) ${ }^{52}$, confirmando un efecto metabólico favorable tras consumir mayor aporte de carbohidratos temprano durante el día.

Esos datos enfatizan que tanto el timing de alimentación como la distribución del aporte de energía y de macronutrientes proveniente de los alimentos, tienen roles importantes en la regulación metabólica. Aún cuando los mecanismos por 
los que esta modificación influye significativamente en la regulación energética no son del todo conocidos, se ha propuesto que la moficación del gasto energético relacionado a la alimentación (termogénesis inducida por la dieta) y la modificación del patrón de expresión de los genes reloj, podrían ser parte de los factores explicativos ${ }^{2,53}$ (Figura 1).

\section{Alteración en el patrón del ciclo sueño-vigilia}

La evidencia anterior respalda la hipótesis que sostiene que una inadecuada temporalidad y calidad de la ingesta de alimentos influye negativamente en el sistema circadiano. Asimismo, el patrón diario ayuno/alimentación es influenciado por el patrón sueño-vigilia, el que a su vez es una de las adaptaciones más evidentes a los RC, con un importante rol en la mantención de un adecuado estado de salud general y metabólica ${ }^{40}$.

Las características del sueño nocturno se han modificado considerablemente en sociedades occidentalizadas, asociado al contexto social de 24 horas, abundante disponibilidad de luz eléctrica, excesiva utilización de aparatos tecnológicos, mayor demanda laboral, turnos laborales y viajes transmeridianos ${ }^{54}$. Asumiendo la alimentación, el sueño y la actividad física como aspectos íntimamente relacionados en la mantención y promoción del estado de salud, no debiera sorprendernos que la alteración o pérdida del orden temporal encamine a la aparición y/o empeoramiento de alteraciones metabólicas tempranas asociadas a obesidad ${ }^{40}$. En efecto, la alteración tanto de la cantidad como la calidad del sueño nocturno ha sido propuesta como factor asociado a obesidad y otras enfermedades crónicas no transmisibles ${ }^{39}$, con estudios recientes confirmando la asociación entre sueño de corta duración y medidas de adiposidad como el IMC o la circunferencia de cintura ${ }^{55}$. La restricción de las horas de sueño se asocia con cambios endocrinos y
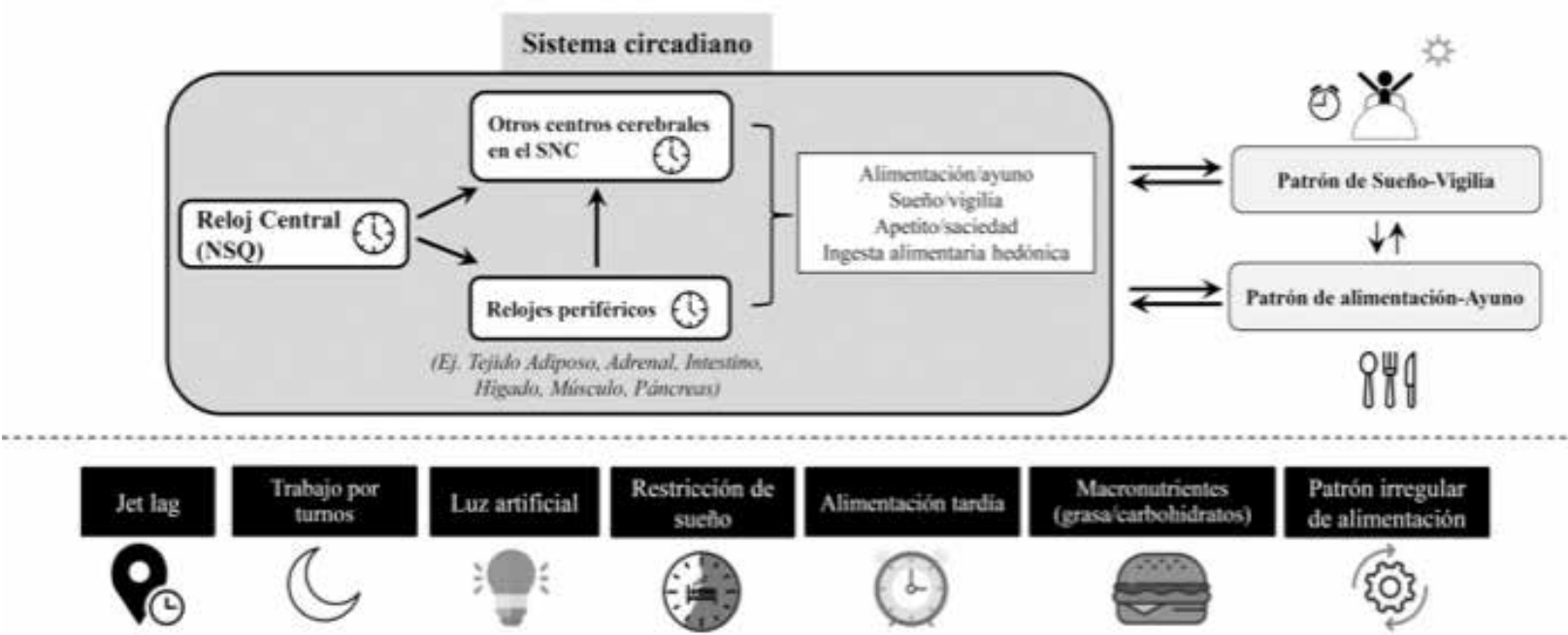

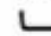
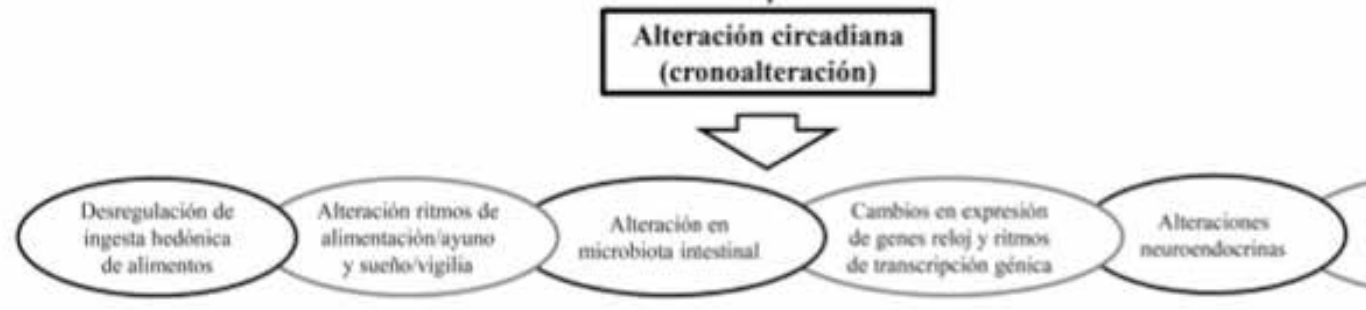

Alternciones

metakolicas de glacosa lipidos

Figura 1. El sistema circadiano y consecuencias metabólicas de la alteración circadiana. El sistema circadiano conformado por el reloj central localizado en el NSQ y diversos relojes u osciladores periféricos a nivel central (fuera del NSQ) y en órganos periféricos, influencia diariamente diversos procesos relacionados al balance energético, entre estos los ritmos de alimentación-ayuno, el ciclo sueño-vigilia (CSV), y la respuesta metabólica a la ingesta de alimentos. Interesantemente, tanto el ritmo de alimentación-ayuno como el CSV tienen un efecto regulador sobre el sistema circadiano. En su conjunto, la influencia circadiana sobre distintos procesos metabólicos determina un orden interno sincronizado diariamente con el medio externo. Diversos factores pueden alterar esta normal regulación circadiana induciendo un desajuste circadiano o cronoalteraciòn. NSQ: núcleo supraquiasmàtico; SNC: sistema nervioso central. 
modificación de la regulación de la ingesta alimentaria, induciendo mayor apetencia por alimentos ricos en energía, un sobreconsumo de energía luego de un mayor tiempo de vigilia, disminución de la actividad física ${ }^{56}$, y afectando negativamente el metabolismo de la glucosa y lípidos ${ }^{57}$. Un aspecto de actual interés resultan sus efectos sobre la regulación hedónica de la ingesta de alimentos y apetencia por alimentos densos en energía ${ }^{56}$. La fragmentación del sueño, íntimamente asociada a una mala calidad del mismo, altera el metabolismo de la glucosa ${ }^{58}$, perfil lipídico, inflamatorio y la regulación cardiovascular. Se reconoce además que la obesidad per se conlleva alteraciones de la organización del sueño, afectando el tono de alerta diurna, aún en ausencia de trastornos respiratorios como la apnea obstructiva del sueño ${ }^{59}$, sugiriendo una interacción bidireccional entre ambos fenómenos que gatillan o intensifican las alteraciones metabólicas.

Los efectos coadyuvantes de la alteración del sueño y de la ritmicidad circadiana alteran la regulación metabólica y aumentan el riesgo de obesidad. Interesantemente, se reconoce que, así como la ritmicidad circadiana influencia la organización del CSV, el patrón de sueño-vigilia también influencia al sistema circadiano y su sincronía es fundamental para una adecuada "salud circadiana". Los efectos negativos de un sueño restringido incluyen la alteración de la expresión de los RC en humanos y de su adecuada influencia sobre genes que regulan la homeostasis metabólica ${ }^{60}$. De hecho, una restricción de sueño moderada así como un sueño desajustado temporalmente (esto es, cuando el sueño ocurre en desincronía del periodo de luz/oscuridad, como en trabajadores por turnos horarios) inducen notables cambios en la expresión rítmica del transcriptoma humano, alterando la normal expresión molecular de los RC y genes relacionados con vías metabólicas e inflamatorias ${ }^{61}$, ilustrando la influencia de un sueño adecuado para la organización circadiana y metabólica (Figura 1).

El denominado jetlag social se define como el desajuste crónico del horario y del tiempo destinado a dormir en la noche entre los días de semana y de fin de semana, reflejando la desincronización temporal similar al fenómeno del jetlag que ocurre en viajes transmeridianos ${ }^{62}$. El jetlag social está fuertemente asociado a obesidad y tendría efecto disímil en individuos de peso normal y obesos, aún controlando estadísticamente por duración y horarios de sueño ${ }^{62}$. También se asocia directamente con circunferencia abdominal, factores del síndrome metabólico y masa grasa ${ }^{63}$, resultados que apoyan que el desajuste entre nuestro reloj biológico y el contexto social, favorece la enfermedad metabólica.

Otro factor asociado a una cronoalteración es la exposición a la luz artificial, donde se ha demostrado que la luz tenue (dim light, en inglés) induce un desajuste circadiano y alteraciones propias del síndrome metabólico y resistencia insulínica en roedores ${ }^{64}$, consitituyendo un aspecto de relevancia en investigación actual sobre el metabolismo de glucosa, apetencia e ingesta energética en humanos, datos que aún no son concluyentes.
Finalmente, el trabajo en modalidad de turnos horarios es una condición particular que, si bien incluye modificación del CSV, se relaciona con una notable alteración circadiana (Figura 1). Alrededor del $15-20 \%$ de la población laboral europea realiza su actividad en turnos horarios, ya sea a horario fijo, rotativo o de cualquier otra modalidad ${ }^{65}$. Este funcionamiento laboral determina la confluencia de alteraciones circadiana, del sueño, del PA y de actividad física ${ }^{66}$. En este subgrupo de la población se ha descrito consistentemente mayor frecuencia de alteraciones gastrointestinales, obesidad, DT2, infarto al miocardio y enfermedad cardiovascular ${ }^{65,67}$. Respecto de la dieta, algunos estudios muestran similar y/o mayor ingesta calórica total en trabajadores de turnos vs. aquellos en horario diurno tradicional, mientras que una mala calidad dietaria y un PA irregular son más concordantes en aquellos en turnos ${ }^{68}$. Una revisión sistemática de estudios observacionales publicados entre 1990-2010, concluyó que los trabajadores por turnos tienen mayor ganancia de peso y prevalencia de obesidad, pero destacó la escasez de datos sobre el PA y recomendación dietaria en este grupo ${ }^{69}$. De hecho, el que la ingesta calórica total no difiera entre los trabajadores nocturnos y diurnos, apunta a que factores otros que el aporte calórico están involucrados en sus efectos metabólicos adversos ${ }^{68}$.

Un desafío actual en este sentido, lo constituye el evaluar el efecto del enfoque nutricional y sus efectos sobre la regulación del peso corporal, la salud metabólica y la funcionalidad en condiciones laborales. Potenciales aspectos de investigación futura debieran evaluar modificaciones específicas de alimentos a consumir a través de las 24 horas, en condiciones laborales y no-laborales, indicación nutricional que debiera incluir el aporte nutricional $y$, particularmente, el timing en que la alimentación debiera ocurrir. Datos bastante recientes y sugerentes ${ }^{26}$, apuntan a que la restricción temporal de la ingesta alimentaria podría influir positivamente en la alteración circadiana y metabólica, aún sin modificar la cantidad y/o calidad de la dieta, lo que pudiera relacionarse con la restauración del ritmo de ayuno/alimentación, mejoramiento del patrón de sueño, eventual reducción de la ingesta calórica, o con cambios en la microbiota intestinal, pues existe evidencia de que la alteración de los RC y una condición de jetlag, altera los ritmos de alimentación-ayuno, y altera la ritmicidad de la microflora intestinal asociado a intolerancia a la glucosa y obesidad ${ }^{70}$.

\section{CONCLUSIÓN}

Los RC influencian fuertemente los procesos y vías metabólicas relacionadas con la alimentación y la nutrición. A su vez, la ingesta de alimentos es un factor regulador del sistema circadiano. La mantención de horarios regulares de alimentación podría constituir un factor importante para una mejor utilización metabólica y estabilidad del peso corporal. Dado que las respuestas metabólicas a la ingesta de alimento difieren a través de las 24 horas, no da lo mismo comer la misma comida, pero en distinto momento del día. 
Las recomendaciones nutricionales debieran considerar e incluir el timing de las comidas, regulando tanto la cantidad (aporte calórico, distribución de macro-y micro-nutrientes) como la calidad de la dieta en el marco temporal de las 24 h. Esto es un desafío actual para ser focalizado en grupos específicos como inviduos con alteraciones metabólicas o en aquellos expuestos a alteración de la ritmicidad circadiana, como los trabajadores en modalidad turnos horarios. Considerando nuestro irremisible marco temporal de 24 horas, resulta evidente la necesidad de promover patrones de ayuno-alimentación, actividad física y sueñovigilia "saludables".

\section{BIBLIOGRAFÍA}

1. Waterhouse J, Minors D, Atkinson G, Benton D. Chronobiology and meal times: Internal and external factors. Br J Nutr 1997; 77(Suppl 1): S29-38.

2. Tahara Y, Shibata S. Chronobiology and nutrition. J Neurosci 2013; 253: 78-88.

3. López AM, Rol de Lama M, Madrid J. Biological rhythms in nutrition and metabolism. In: Madrid J, De Lama M, editors. Basical and clinical chronobiology. 1st ed. Madrid: Editec@ red; 2006. p. 513-552.

4. Reppert SM, Weaver DR. Coordination of circadian timing in mammals. Nature. 2002/08/29. 2002; 418(6901): 935-941.

5. Dardente H, Dardente H, Cermakian N. Molecular Circadian Rhythms in Central and Peripheral Clocks in Mammals. Chronobiol Int 2007;24(2): 195-213.

6. Stratmann M, Schibler U. Properties, Entrainment, and Physiological Functions of Mammalian Peripheral Oscillators. J Biol Rhythms 2006; 21(6): 494-506.

7. Asher G, Sassone-Corsi P. Time for food: The intimate interplay between nutrition, metabolism, and the circadian clock. Cell 2015; 161(1): 84-92.

8. Hattar S, Lucas RJ, Mrosovsky N, Thompson S, Douglas RH, Hankins MW, et al. Melanopsin and rod-cone photoreceptive systems account for all major accessory visual functions in mice. Nature 2003; 424(6944): 76-81.

9. Albrecht U. Timing to Perfection: The Biology of Central and Peripheral Circadian Clocks. Neuron 2012; 74(2): 246-260.

10. Husse J, Eichele G, Oster H. Synchronization of the mammalian circadian timing system: Light can control peripheral clocks independently of the SCN clock: Alternate routes of entrainment optimize the alignment of the body's circadian clock network with external time. BioEssays 2015; 37(10): 1119-1128.

11. Eckel-Mahan K, Sassone-Corsi P. Metabolism and the Circadian Clock Converge. Am Physiol Soc 2013; 93(1): 107-135.

12. Scheer F a JL, Morris CJ, Shea S a. The internal circadian clock increases hunger and appetite in the evening independent of food intake and other behaviors. Obesity (Silver Spring) 2013 Mar; 21(3): 421-423.

13. Konturek PC, Brzozowski T, Konturek SI. Gut clock: Implication of circadian rhythms in the gastointestinal tract. J Physiol Pharmacol 2011; 62(2): 139-150.

14. Hatori $M$, Vollmers C, Zarrinpar A, DiTacchio L, Bushong EA, Gill S, et al. Time-restricted feeding without reducing caloric intake prevents metabolic diseases in mice fed a high-fat diet. Cell Metab 2012; 15(6): 848-860.

15. Leech RM, Worsley A, Timperio A, McNaughton SA. Understanding meal patterns: definitions, methodology and impact on nutrient intake and diet quality. Nutr Res Rev 2015;
1-21.

16. de Castro JM. Circadian rhythms of the spontaneous meal pattern, macronutrient intake, and mood of humans. Physiol Behav 1987; 40(4): 437-446.

17. Affenito SG. Breakfast: a missed opportunity. I Am Diet Assoc 2007; 107(4): 565-569.

18. Toschke AM, Thorsteinsdottir KH, von Kries R. Meal frequency, breakfast consumption and childhood obesity. Int J Pediatr Obes 2009; 4(December 2008): 242-248.

19. Farshchi HR, Taylor MA, Macdonald IA. Deleterious effects of omitting breakfast on insulin sensitivity and fasting lipid profiles in healthy lean women. Am J Clin Nutr 2005; 81(2): 388-396.

20. Betts JA, Richardson ID, Chowdhury EA, Holman GD, Tsintzas $K$, Thompson $D$. The causal role of breakfast in energy balance and health: A randomized controlled trial in lean adults. Am J Clin Nutr 2014; 100(2): 539-547.

21. Toschke, A., Küchenhoff, H., Koletzko, B. \& Von Kries R. Meal Frequency and Childhood Obesity. Obes Res 2005; 13(11): 1932-1938.

22. Stote KS, Baer DJ, Spears K, Paul DR, Harris GK, Rumpler W $V$., et al. A controlled trial of reduced meal frequency without caloric restriction in healthy, normal-weight, middle-aged adults. Am J Clin Nutr 2007; 85(4): 981-988.

23. Farshchi HR, Taylor MA, Macdonald IA. Beneficial metabolic effects of regular meal frequency on dietary thermogenesis, insulin sensitivity, and fasting lipid profiles in healthy obese women. Am J Clin Nutr 2005; 81(1): 16-24.

24. Leidy HJ, Campbell WW. The Effect of Eating Frequency on Appetite Control and Food Intake: Brief Synopsis of Controlled Feeding Studies. I Nutr 2011; 141: 154-157.

25. Varady KA. Meal frequency and timing: impact on metabolic disease risk. Curr Opin Endocrinol Diabetes Obes 2016; 23(5): 379-383.

26. Gill S, Panda S. A Smartphone App Reveals Erratic Diurnal Eating Patterns in Humans that Can Be Modulated for Health Benefits. Cell Metab 2015; 22(5): 789-798.

27. Mattson MP, Allison DB, Fontana L, Harvie M, Longo VD, Malaisse WJ, et al. Meal frequency and timing in health and disease. Proc Natl Acad Sci 2014; 111(47): 16647-16653.

28. Shukla AP, Andono I, Touhamy SH, Casper A, Iliescu RG, Mauer $E$, et al. Carbohydrate-last meal pattern lowers postprandial glucose and insulin excursions in type 2 diabetes. BMJ Open Diabetes Res Care 2017; 5(1): e000440.

29. Damiola F, Le Minli N, Preitner N, Kornmann B, FleuryOlela F, Schibler U. Restricted feeding uncouples circadian oscillators in peripheral tissues from the central pacemaker in the suprachiasmatic nucleus. Genes Dev 2000; 14(23): 2950-2961.

30. Petersen CC, Patton DF, Parfyonov M, Mistlberger RE. Circadian food anticipatory activity: Entrainment limits and scalar properties re-examined. Behav Neurosci 2014; 128(6): 689-702.

31. Mistlberger RE. Neurobiology of food anticipatory circadian rhythms. Physiol Behav 2011; 104(4): 535-545.

32. Arble DM, Bass J, Laposky AD, Vitaterna MH, Turek FW. Circadian timing of food intake contributes to weight gain. Obesity (Silver Spring). 2009/09/05. 2009; 17(11): 2100-2102.

33. Bray M, Tsai J-Y, Villegas-Montoya C, Boland B, Blasier $Z$, Egbejimi $O$, et al. Time-of-day-dependent dietary fat consumption influences multiple cardiometabolic syndrome parameters in mice. Int J Obes 2010; 34(10): 1589-1598.

34. Sherman H, Genzer Y, Cohen R, Chapnik N, Madar Z, Froy 
O. Timed high-fat diet resets circadian metabolism and prevents obesity. FASEB J 2012 Aug; 26(8): 3493-3502.

35. Garaulet M, Gómez-Abellán P, Alburquerque-Béjar JJ, Lee Y-C, Ordovás JM, Scheer FA. Timing of food intake predicts weight loss effectiveness. Int J Obes (Lond) 2013; 37(4): 604-611.

36. Jakubowicz D, Wainstein J, Ahrén B, Bar-Dayan Y, Landau $Z$, Rabinovitz HR, et al. High-energy breakfast with lowenergy dinner decreases overall daily hyperglycaemia in type 2 diabetic patients: a randomised clinical trial. Diabetologia 2015; 58(5): 912-919.

37. Moro T, Tinsley G, Bianco A, Marcolin G, Pacelli QF, Battaglia $G$, et al. Effects of eight weeks of time-restricted feeding (16/8) on basal metabolism, maximal strength, body composition, inflammation, and cardiovascular risk factors in resistance-trained males. J Trans/ Med 2016; 14(1): 290.

38. World Health Organization. Diet, nutrition and the prevention of chronic diseases. World Heal Organ Tech Rep Ser 2003; 916 i-viii-1-149.

39. Chamorro RA, Durán SA, Reyes SC, Ponce R, Algarín CR, Peirano $P D$. The reduction of sleep as a risk factor for obesity. Rev Med Chil 2011; 139(7): 932-940.

40. Peirano P, Algarín C, Díaz E, Garrido M, Sir T, Albala C. Sleep/wake cycle and obesity. Rev Chil Nutr 2000;(1): 1-12.

41. Kant AK, Graubard BI. 40-Year Trends in Meal and Snack Eating Behaviors of American Adults. I Acad Nutr Diet 2015; 115(1): 50-63.

42. Gupta NJ, Kumar V, Panda S. A camera-phone based study reveals erratic eating pattern and disrupted daily eatingfasting cycle among adults in India. PLoS One 2017; 12(3): 1-15.

43. Baron KG, Reid KJ, Kern AS, Zee PC. Role of sleep timing in caloric intake and BMI. Obesity (Silver Spring). 2011; 19(7): 1374-1381.

44. Wang JB, Patterson RE, Ang A, Emond JA, Shetty N, Arab L. Timing of energy intake during the day is associated with the risk of obesity in adults. J Hum Nutr Diet 2014; 27(S2): 255-262.

45. Westerterp-Plantenga $M$, IJedema $M$, Wijckmans-Duijsens $N$. The role of macronutrient selection in determining patterns of food intake in obese and non-obese women. Eur J Clin Nutr. 1996; 50(9): 580-591.

46. McHill AW, Phillips AJ, Czeisler CA, Keating L, Yee K, Barger $L K$, et al. Later circadian timing of food intake is associated with increased body fat. Am J Clin Nutr 2017; ajcn161588.

47. Jakubowicz D, Barnea M, Wainstein J, Froy O. High Caloric intake at breakfast vs. dinner differentially influences weight loss of overweight and obese women. Obesity (Silver Spring) Dec; 21(12): 2504-2512.

48. Almoosawi S, Prynne CJ, Hardy R, Stephen AM. Diurnal eating rhythms: Association with long-term development of diabetes in the 1946 British birth cohort. Nutr Metab Cardiovasc Dis 2013; 23(10): 1025-1030.

49. Baron KG, Reid KJ, Horn L Van, Zee PC. Contribution of evening macronutrient intake to total caloric intake and body mass index. Appetite 2013; 60(1): 246-251.

50. Jakubowicz D, Froy O, Wainstein J, Boaz M. Meal timing and composition influence ghrelin levels, appetite scores and weight loss maintenance in overweight and obese adults. Steroids 2012; 77(4): 323-331.

51. Sofer S, Eliraz A, Kaplan S, Voet H, Fink G, Kima T, et al. Greater weight loss and hormonal changes after 6 months diet with carbohydrates eaten mostly at dinner. Obesity
(Silver Spring). 2011; 19(10): 2006-2014.

52. Kessler K, Hornemann S, Petzke KJ, Kemper M, Kramer A, Pfeiffer AFH, et al. The effect of diurnal distribution of carbohydrates and fat on glycaemic control in humans: a randomized controlled trial. Sci Rep 2017; 7: 44170.

53. Johnston ID, Scheer FA, Turek FW. Circadian Rhythms, Metabolism, and Chrononutrition in Rodents and Humans. Adv Nutr 2016; 399-406.

54. Rajaratnam SMW, Arendt J. Health in a 24-h society 2001; 358: 999-1005.

55. Sperry SD, Scully ID, Gramzow RH, Jorgensen RS. Sleep Duration and Waist Circumference in Adults: A MetaAnalysis. Sleep 2015; 38(8): 1269-1276.

56. Schmid SM, Hallschmid M, Schultes B. The metabolic burden of sleep loss. Lancet Diabetes Endocrinol 2015 Jan; 3(1): 52-62.

57. Broussard IL, Chapotot F, Abraham V, Day A, Delebecque $F$, Whitmore $H R$, et al. Sleep restriction increases free fatty acids in healthy men. Diabetologia 2015; 58(4): 791-798.

58. Stamatakis KA, Punjabi NM. Effects of sleep fragmentation on glucose metabolism in normal subjects. Chest 2010; 137(1): 95-101.

59. Vgontzas a N, Bixler EO, Tan TL, Kantner D, Martin LF, Kales a. Obesity without sleep apnea is associated with daytime sleepiness. Arch Intern Med. 1998; 158: 1333-1337.

60. Archer SN, Oster H. How sleep and wakefulness influence circadian rhythmicity: Effects of insufficient and mistimed sleep on the animal and human transcriptome. I Sleep Res 2015; 24(5): 476-493.

61. Archer $S N$, Laing EE, Möller-Levet $C S$, van der Veen $D R$, Bucca G, Lazar AS, et al. Mistimed sleep disrupts circadian regulation of the human transcriptome. Proc Natl Acad Sci 2014; 111(6): E682-E691.

62. Roenneberg T, Allebrandt K V., Merrow M, Vetter C. Social jetlag and obesity. Curr Biol 2012; 22(10): 939-943.

63. Parsons MI, Moffitt TE, Gregory AM, Goldman-Mellor $S$, Nolan PM, Poulton $R$, et al. Social jetlag, obesity and metabolic disorder: investigation in a cohort study. Int J Obes 2015; 39(5): 842-848.

64. Fonken LK, Workman IL, Walton JC, Weil ZM, Morris JS, Haim A, et al. Light at night increases body mass by shifting the time of food intake. Proc Natl Acad Sci 2010; 107(43): 18664-1869.

65. Boivin DB, Tremblay GM, James FO. Working on atypical schedules. Sleep Med 2007; 8(6): 578-589.

66. Broussard IL, Van Cauter E. Disturbances of sleep and circadian rhythms: novel risk factors for obesity. Curr Opin Endocrinol Diabetes Obes 2016; 23(5): 353-359.

67. Haupt CM, Alte D, Dörr M, Robinson DM, Felix SB, John $U$, et al. The relation of exposure to shift work with atherosclerosis and myocardial infarction in a general population. Atherosclerosis 2008; 201(1): 205-211.

68. Lowden A, Moreno C, Holmbäck U, Lennernäs M, Tucker $P$. Eating and shift work - Effects on habits, metabolism, and performance. Scand J Work Environ Heal 2010; 36(2): 150-162.

69. Amani R, Gill T. Shiftworking, nutrition and obesity: Implications for workforce health- a systematic review. Asia Pac J Clin Nutr 2013; 22: p. 505-515.

70. Thaiss CA, Zeevi D, Levy M, Zilberman-Schapira G, Suez J, Tengeler AC, et al. Transkingdom control of microbiota diurnal oscillations promotes metabolic homeostasis. Cell 2014; 159(3): 514-529. 